\title{
Poorly Differentiated Neuroendocrine Carcinoma of the Parotid Gland and Moderately Differentiated Hepatic Metastases: A Discordant Histopathology Clarified by Dual-Tracer PET/CT
}

\author{
Priyanka Sharma and Sandip Basu \\ Radiation Medicine Centre, Bhabha Atomic Research Centre, Tata Memorial Centre Annexe, and Homi Bhabha National Institute, \\ Mumbai, India
}

\begin{abstract}
This teaching case study illustrates a metastatic neuroendocrine neoplasm (NEN) of the parotid gland in which the histopathologic findings were discordant between the primary parotid tumor (poorly differentiated small cell neuroendocrine carcinoma) and a large hepatic metastasis (atypical carcinoid with moderately differentiation status; Ki-67, 15\%-20\%). The case study also illustrates the value of dual-tracer PET/CT imaging ( ${ }^{68} \mathrm{Ga}-\mathrm{DOT} A T A T E$ and $\left.{ }^{18} \mathrm{~F}-\mathrm{FDG}\right)$ in such a clinical setting. Minimal uptake of ${ }^{68} \mathrm{Ga}$-DOTATATE and high-grade uptake of ${ }^{18} \mathrm{~F}-\mathrm{FDG}$ in the lesions indicated a poor differentiation status and helped to clarify the tumor biology, with potential implications for subsequent treatment-decision individualization in favor of chemotherapy. The findings underscore the clinical utility of dual-tracer PET/CT in making the appropriate assessment in patients with conflicting or discordant histopathologic findings at 2 sites.
\end{abstract}

Key Words: head and neck; ${ }^{18} \mathrm{~F}-\mathrm{FDG}$ PET/CT; neuroendocrine tumor; oncology; parotid gland; ${ }^{68 \mathrm{Ga}-D O T A T A T E}$

J Nucl Med Technol 2021; 49:86-88

DOI: $10.2967 /$ jnmt.120.251587

\section{O}

mall cell neuroendocrine carcinoma of the salivary glands accounts for about $2 \%$ of all salivary gland malignancies and is the commonest form of salivary gland neuroendocrine neoplasm (NEN), usually arising in the parotid glands. Histologically, small cell neuroendocrine carcinoma is very similar to small cell carcinoma of the lung and is composed of tumor cells arranged in solid diffuse sheets or ribbons and other patterns. Well-differentiated NENs of the salivary gland are exceedingly rare. To our knowledge, only 7 cases have been reported; these include

\footnotetext{
Received Jun. 14, 2020; revision accepted Aug. 7, 2020.

For correspondence or reprints contact: Sandip Basu, Radiation Medicine Centre, Bhadha Atomic Research Centre, Tata Memorial Centre Annexe, Parel, Mumbai 400012, India.

E-mail: drsanb@yahoo.com

Published online Oct. 5, 2020

COPYRIGHT (C) 2021 by the Society of Nuclear Medicine and Molecular Imaging.
}

2 reports of typical carcinoid and 5 of atypical carcinoid (1), all occurring in the parotid glands.

Akin to what is observed in gastroenteropancreatic NENs, identifying predictors of tumor biology is of substantial importance in salivary gland NENs to predict the clinical behavior of the tumor and guide appropriate treatment strategies. Determining the grade often relies on examining the previously resected primary tumor or a biopsy sample of a single metastatic lesion that may not be consistent with the primary, reflecting tumor heterogeneity and biology $(2,3)$. In a typical case scenario, the lowergrade NENs should show high somatostatin receptor expression (imaged on ${ }^{68} \mathrm{Ga}$-DOTATATE PET/CT) more than glucose transporter expression and metabolism (on ${ }^{18} \mathrm{~F}-$ FDG PET/CT), and vice versa with poorly differentiated neoplasms $(2,3)$.

\section{CASE REPORT}

A 39-y-old woman, diagnosed with a malignant round cell neoplasm of the right parotid gland on biopsy, had undergone right radical parotidectomy 2 y previously. The final histopathologic findings were suggestive of poorly differentiated NEN (small cell variant), with positive inked margins. Postoperatively, she received adjuvant radiotherapy and 4 cycles of systemic chemotherapy (carboplatinetoposide regimen). At the 2-y follow-up, there was an isoechoic space-occupying lesion $(7.4 \times 6.1 \mathrm{~cm})$ in the right lobe of the liver on ultrasonography (abdominal), confirmed by triple-phase contrast-enhanced CT with additional mention of a large area of portocaval lymphadenopathy. Liver biopsy indicated a moderately differentiated carcinoid tumor. The immunohistochemistry supported a neuroendocrine origin; was positive for synaptophysin, chromogranin, and cytokeratin; was negative for hepatocyte-specific antigen (or hepatocyte paraffin 1); and indicated a Ki-67 of $15 \%-$ $20 \%$-features compatible with an atypical carcinoid of World Health Organization grade 2. 


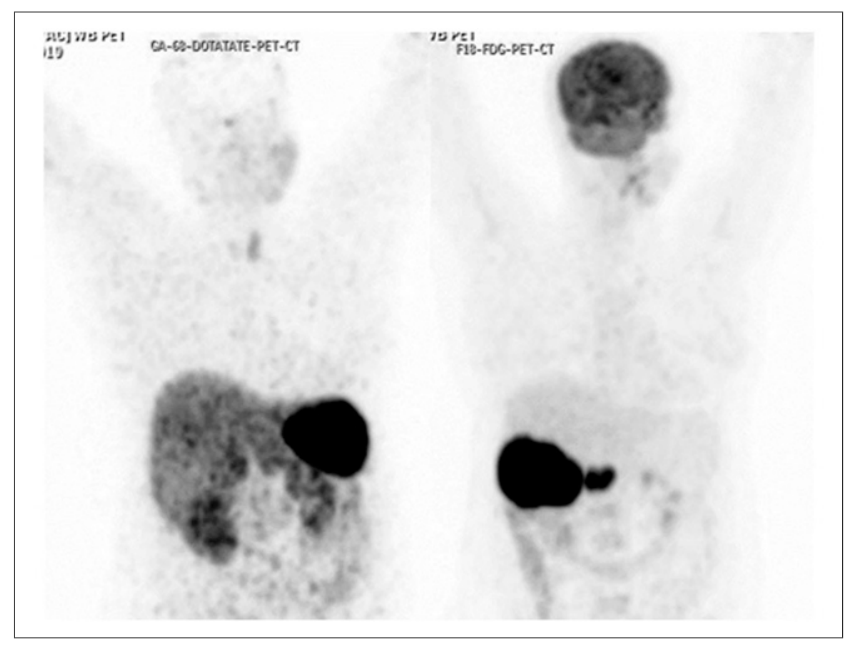

FIGURE 1. ${ }^{68} \mathrm{Ga}-\mathrm{DOTATATE}$ (left) and ${ }^{18} \mathrm{~F}-\mathrm{FDG}$ (right) PET/CT maximum-intensity-projection images demonstrating high uptake of ${ }^{18} \mathrm{~F}-\mathrm{FDG}$ in metastatic liver lesion and portocaval lymphadenopathy but low or minimal uptake of somatostatin receptor-based ${ }^{68} \mathrm{Ga}-\mathrm{DOTATATE}$.

A dual-tracer $\left({ }^{68} \mathrm{Ga}\right.$-DOTATATE and $\left.{ }^{18} \mathrm{~F}-\mathrm{FDG}\right) \mathrm{PET} / \mathrm{CT}$ evaluation before treatment decision making demonstrated intense ${ }^{18}$ F-FDG uptake in a liver space-occupying lesion (SUV $\left._{\max }, 23.92\right)$ and in the portocaval lymph nodes $\left(\mathrm{SUV}_{\max }\right.$, 16.9) but minimal ${ }^{68} \mathrm{Ga}$-DOTATATE avidity, suggestive of a low somatostatin receptor expression compatible with poorly differentiated neuroendocrine carcinoma, rather than a moderately differentiated atypical carcinoid (Figs. 1 and
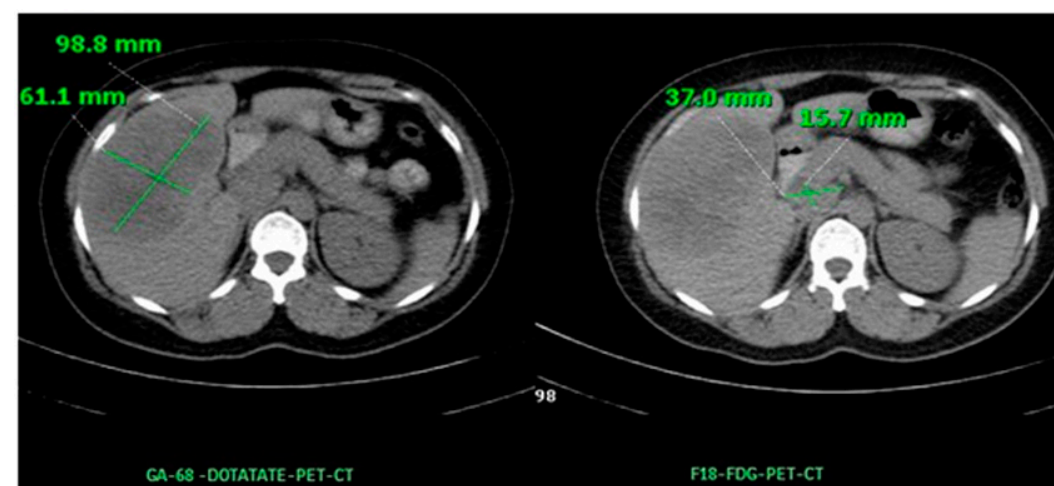

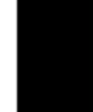

GA-68 - DOTATATE-PET-CT

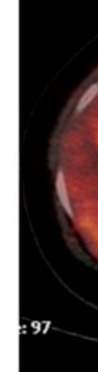

97

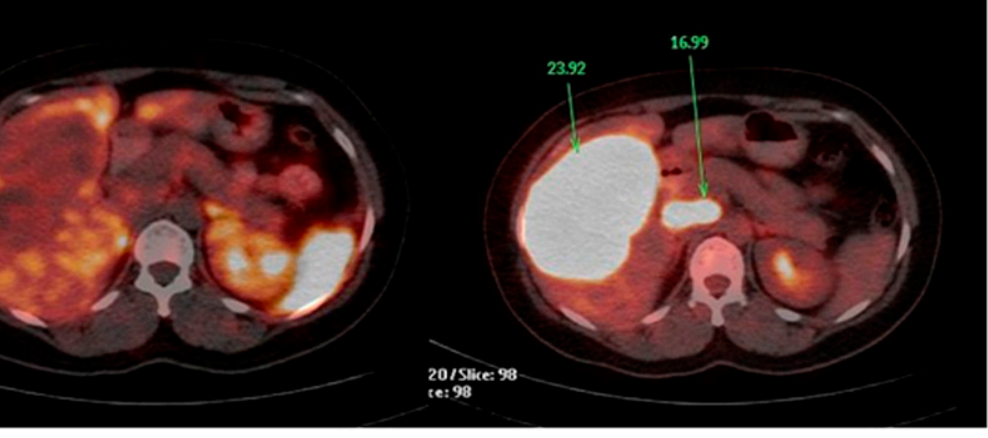

FIGURE 2. Axial CT (top) and PET/CT (bottom) images demonstrating limited ${ }^{68} \mathrm{Ga}-$ DOTATATE uptake in metastatic liver lesion (left) and portocaval lymph nodes but prominent ${ }^{18} \mathrm{~F}$-FDG uptake (right).
2). The scan findings thus helped in clarifying the tumor biology, as is important in such cases in which histopathologic findings show a conflict between 2 sites and are not sufficient alone.

\section{DISCUSSION}

A relatively rare entity, NENs of the head and neck region pose diagnostic and therapeutic challenges. With the availability of multimodal treatment and the recently developed peptide receptor radionuclide therapy for metastatic NENs, proper selection of patients for a particular treatment is important to the subsequent management. The Ki-67 index is useful for predicting tumor biology and metastasis and for deciding the multimodal approach toward therapy. Regarding tumor aggressiveness, poorly differentiated NENs are the most aggressive, followed by atypical carcinoids (moderately differentiated) and then typical carcinoids; this stratification has implications for assigning the appropriate treatment. In the present case, low or minimal ${ }^{68} \mathrm{Ga}$-DOTATATE uptake and high-grade ${ }^{18} \mathrm{~F}$ FDG uptake in the lesions (despite the reported Ki-67 of $15 \%-20 \%$ on liver biopsy) favored poorly differentiated or G3 tumor, the usual characteristic of this subtype. This possibility was commensurate with the commonly observed spectrum of salivary gland NENs_-primarily poorly differentiated neuroendocrine carcinomas of small cell and large cell types - with very few cases of well-differentiated NENs (carcinoids) being reported $(2,3)$.

Tumor $\mathrm{SUV}_{\max }$ on somatostatin receptor-based PET is predictive of response to somatostatin receptor-targeted therapies such as peptide receptor radionuclide therapy, whereas high ${ }^{18} \mathrm{~F}$ FDG uptake has been considered the basis for choosing chemotherapy. Dualtracer PET/CT is thus potentially helpful for such tumor stratification and treatment decision making. In the described patient, the tumor biology as evident from dual-tracer PET/CT was consistent with the histopathology of the primary tumor (i.e., small cell neuroendocrine carcinoma of the parotid gland), and chemotherapy was considered preferred over peptide receptor radionuclide therapy.

\section{CONCLUSION}

Dual-tracer PET/CT has clinical utility in making the appropriate assessment in patients with conflicting or discordant histopathologic findings at 2 sites. 


\section{DISCLOSURE}

No potential conflict of interest relevant to this article was reported.

\section{REFERENCES}

1. Said-Al-Naief N, Sciandra K, Gnepp DR. Moderately differentiated neuroendocrine carcinoma (atypical carcinoid) of the parotid gland: report of three cases with contemporary review of salivary neuroendocrine carcinomas. Head Neck Pathol. 2013;7:295-303.

2. Yang Z, Tang LH, Klimstra DS. Effect of tumor heterogeneity on the assessment of Ki67 labeling index in well-differentiated neuroendocrine tumors metastatic to the liver: implications for prognostic stratification. Am J Surg Pathol. 2011;35: 853-860.

3. Basu S, Ranade R, Ostwal V, Shrikhande SV. PET-based molecular imaging in designing personalized management strategy in gastroenteropancreatic neuroendocrine tumors. PET Clin. 2016;11:233-241. 